\title{
Incompatibility of published ac magnetic susceptibility of a room temperature superconductor with measured raw data
}

\author{
J. E. Hirsch \\ Department of Physics, University of California, San Diego, La Jolla, CA 92093-0319
}

Room temperature superconductivity has recently been reported for a carbonaceous sulfur hydride (CSH) under high pressure by Snider et al [1]. The paper reports sharp drops in magnetic susceptibility as a function of temperature for five different pressures, that are interpreted as signaling a superconducting transition. Here I question the validity and faithfulness of the magnetic susceptibility data presented in the paper by comparison with the measured raw data reported by two of the authors of ref. [1] in ref. [2]. This casts doubt on the assertion of the paper [1] that the susceptibility measurements support the case for superconductivity in this compound.

Ac magnetic susceptibility is a useful measurement to detect the existence of superconductivity in materials under high pressure [3-5]. Because of the smallness of the sample required by the geometry of the diamond anvil cell, the detected signal is a small drop in a large signal coming from the superposition of the sample and the background magnetic susceptibilities, according to the relation

$$
\text { data }=\text { raw data - background signal. }
$$

Ref. [1] states that a background signal determined from a non-superconducting CSH sample at 108 GPa was subtracted from the measured raw data.

The published susceptibility data for pressure $189 \mathrm{GPa}$ (Fig. 2a of ref. [1]) are shown on the left panel of Fig. 1. On the right panel of Fig. 1 I show an expanded form of that graph, obtained from the figure published on-line [1]. Because it is is a vector graphics figure, it allows extraction of the numerical values used for the plot using software such as adobe illustrator and inkscape.

The rectangle on the top right of Fig. 1 encloses a region that is blown up to show it with more resolution in Fig. 2. The right panel of Fig. 2 shows our numbers, and the horizontal red lines connect points that are identical to 6 digit accuracy. In ref. [6] I pointed out that these coincidences appear to be anomalous, and that their significance could possibly be evaluated once the authors of ref. [1] released the raw data and background signal measured and used to construct Fig. 1 according to Eq. (1). I had requested those data from the authors on November 12, 2020, and repeatedly thereafter.

On December 1, 2021, two of the authors of ref. [1] posted a paper on arxiv [2] where for the first time they released the raw data corresponding to the measurements of Fig. 1 (as well as raw data for other pressures). They did not however release the measured values of the background signal that were used to obtain Fig. 1 according to Eq. (1).

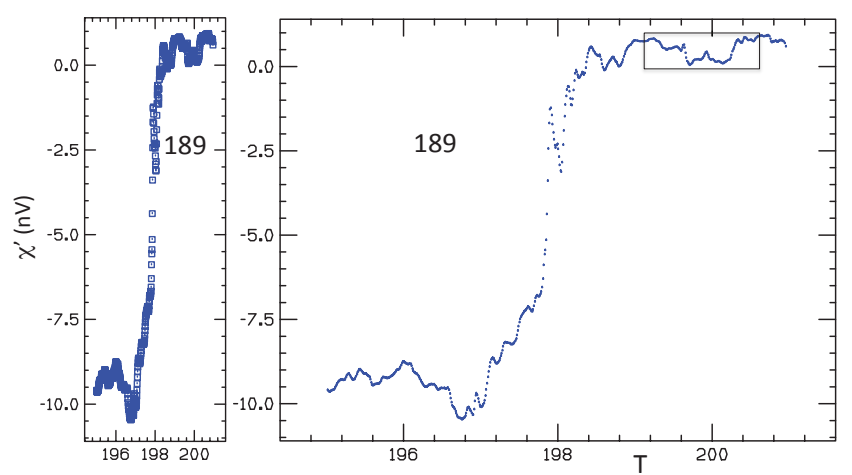

FIG. 1: Magnetic susceptibility for pressure 189 GPa obtained from the vector image of the published Fig. 2a [1]. The left panel shows the data plotted with the same resolution as in Fig. 2a of ref. [1], the right panel with a higher resolution. The rectangle in the right panel shows the region shown with even more resolution in Figs. 2 and 3.

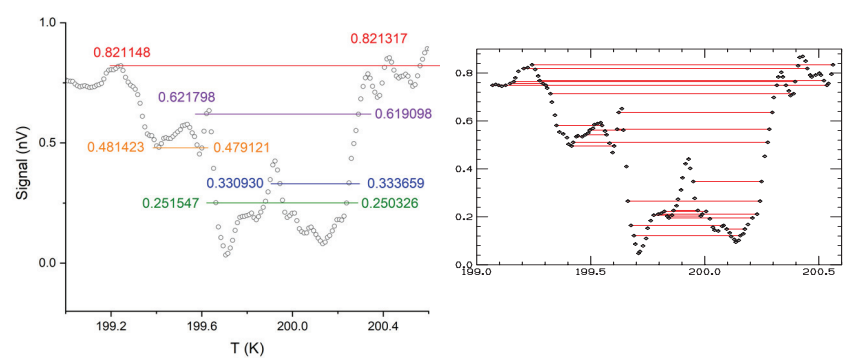

FIG. 2: Right panel: small portion of the curve in Fig. 1 (enclosed in the rectangle in Fig. 1) plotted with higher resolution. The red lines connect values of magnetic susceptibility that are identical to 6 decimal places. Left panel: curve for the same region of temperature published in Fig. 8 of ref. [2].

In that paper, the authors also asserted that their Fig. 2a of [1] that contains the curve shown in Fig. 1 is a raster rather than a vector image, that does not allow to extract numerical values of the data to 6 digits accuracy. Instead, they reported their values for the data for several points of that portion of the curve as shown on the left panel of Fig. 2, arguing that the coincidences pointed out in ref. [6] are within at most 2 digits rather than 6 digits.

I am certain that Fig. 2a of ref. [1] is a vector image and that the data extracted from it are accurate to 6 digits, hence that the authors of [2] are mistaken in their assertion. Be that as it may however, it is not necessary to settle that point here. Irrespective of how it is settled, the importance of Fig. 2 is that the left and right panels 


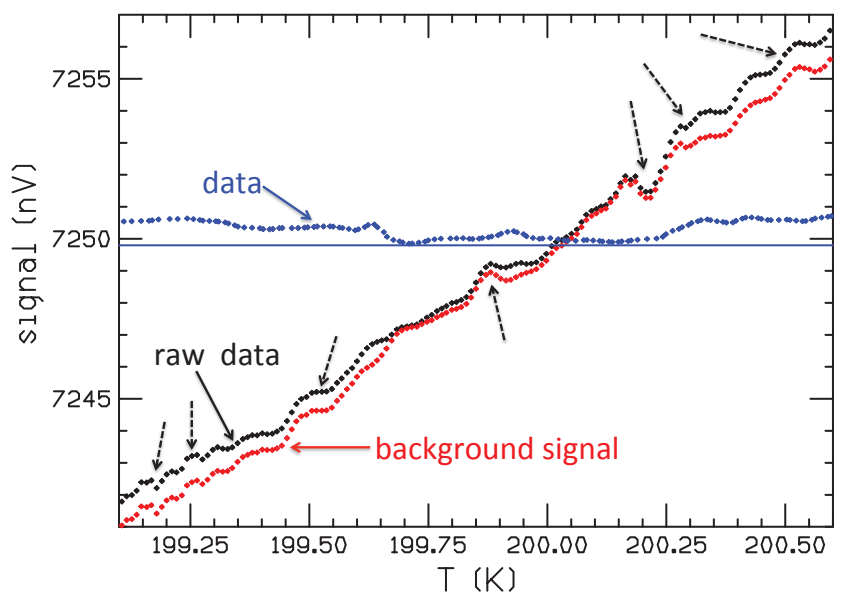

FIG. 3: Raw data from ref. [2] (black points), data from the published Fig. 2a of ref. [1] or equivalently from Fig. 8 of ref. [2] (blue points), and background signal obtained from Eq. (2) (red points). The dashed arrows point to similar fine structure in the raw data and background signal that is not seen in the data.

'look' essentially identical. That means that the authors and I agree that the numerical values of the data that I extracted from the published Figure 2a of [1] faithfully describe the fine structure of their measured curve, that is not discernable with the bare eye from the published figure (left panel of fig. 1).

Given that, and that the authors have so far declined to release the measured background signal, I calculated the background signal from the equation

$$
\text { background signal }=\text { raw data }- \text { data }
$$

derived from Eq. (1), using the raw data in [2] and the published data in [1]. Fig. 3 shows the raw data, published data and background signal obtained from Eq. (2), for the same temperature range shown in Fig. 2.

The significance of Fig. 3 is that the raw data and background signal show fine structure that is not con- tained in the data, as can be clearly seen in several parts of the curves, which I point out with dashed arrows. That structure is presumably due to random noise. However, the raw data and background signal were two independent measurements for two very different pressure values (189 GPa and $108 \mathrm{GPa}$ respectively), so I argue that it is impossible that they could show the same detailed features seen in Fig. 3.

Examination of the raw data and background signal for $189 \mathrm{GPa}$ for the entire temperature range shown in Fig. 1, as well as for the two other values of the pressure reported in Fig. 2a of ref. [1], shows the same similar behavior in wiggles of raw data and background signal displayed in Fig. 3, ubiquitously [7]. It is also noteworthy that the raw data of ref. [2] do not show [7] the anomalous 6-digit coincidences shown in the right panel of Fig. 2 pointed out in ref. [6].

I argue that this provides conclusive proof that either:

(1) The raw data released in ref. [2] are not the real raw data underlying the published susceptibility results in ref. [1], i.e. there are other raw data that have not been released.

(2) The published data in ref. [1] were not obtained by subtracting from the raw data an independently measured background signal at a much lower pressure, but were obtained by some other undisclosed procedure.

Either of these two possibilities calls into serious question the validity of the reported results for ac magnetic susceptibility of CSH in ref. [1]. As a consequence, they call into serious question the claim that CSH under pressure is a room temperature superconductor. Other reasons to question that claim were reported in refs. [8], [9] and [10].

\section{Acknowledgments}

I am grateful to James Hamlin and Kevin Smith for educating me on and helping me with obtaining numerical data from vector graphics images.
[1] E. Snider et al., 'Room-temperature superconductivity in a carbonaceous sulfur hydride', Nature 586, 373 (2020).

[2] Ranga P. Dias and Ashkan Salamat, "Standard Superconductivity in Carbonaceous Sulfur Hydride", arXiv:2111.15017, Dec. 1, 2021.

[3] Y. A. Timofeev et al, Rev. Sci. Inst. 73, 371 (2002).

[4] D. D. Jackson et al, Rev. Sci. Inst. 7374, 2467 (2003).

[5] J. J. Hamlin, "Superconductivity studies at extreme pressure", Washington University, 2007.

[6] J. E. Hirsch, "On the ac magnetic susceptibility of a room temperature superconductor: anatomy of a probable scientific fraud", Physica C 26 September 2021, 1353964 (temporarily removed).

[7] J. E. Hirsch, "Disconnect between published ac mag- netic susceptibility of a room temperature superconductor and measured raw data", preprints.org $>$ physical sciences $>$ condensed matter physics $->$ doi: 10.20944/preprints202112.0115.v1 (2021).

[8] J. E. Hirsch and F. Marsiglio, "Unusual width of the superconducting transition in a hydride", Nature 596, E9, (2021).

[9] M. Dogan and M. L. Cohen, "Anomalous behavior in high-pressure carbonaceous sulfur hydride", Physica C 583, 1353851 (2021).

[10] T. Wang et al, "Absence of conventional room temperature superconductivity at high pressure in carbon doped $H_{3} S^{\prime \prime}$, Phys. Rev. B 104, 064510 (2021). 